\title{
Cavitation développée \\ sur des parois à courbure continue : physique du détachement ${ }^{*}$
}

\section{The cavitation developing on continuous-curve walls: the physics of detachment}

\section{J. M. Michel,} Chargé de Recherche
au C.N.R.S.*

\author{
S. Biset,
}

Chercheur $3^{\mathrm{e}}$ Cycle

\section{J. P. Franc,}

Attaché de Recherche

au C.N.R.S.* *
La théorie classique des écoulements cavitants se fonde sur le schéma hydrodynamique simplifié du fluide non visqueux en écoulement irrotationnel. Prenant en compte les seules forces de gravité, d'inertie et de pression, elle accorde un rôle prééminent au nombre de cavitation $\sigma_{v}$ qui rapporte l'écart entre la pression de référence et la tension de vapeur de l'eau à une pression caractéristique, par exemple la pression dynamique correspondant à une vitesse typique de l'écoulement.

Si l'on traite alors des conditions limites d'apparition de la cavitation, on trouve que la cavitation doit apparaître à l'endroit où la pression est minimale : la valeur $\sigma_{v i}$ de $\sigma_{v}$ à l'apparition de la cavitation doit être égale à la valeur absolue du coefficient de pression $C_{p m}$ en cet endroit.

Dans le cas d'une cavité qui se développe au voisinage d'une paroi solide à courbure continue, l'enveloppe de la cavité se détache, suivant la condition mathématique de Villat [1], de sorte que sa tangente et sa courbure soient en continuité avec celle de la paroi.

Une telle analyse, bien qu'utile pour effectuer des calculs de type global sur le comportement des ailes hydrodynamiques, est évidemment trop pauvre par rapport à la physique réelle pour permettre une prévision correcte des circonstances d'apparition et de développement de la cavitation. De longue date on a d'ailleurs relevé des comportements singuliers, voire aberrants, par rapport aux prévisions de la théorie hydrodynamique : différence entre la valeur réelle du coefficient $\sigma_{v i}$ d'apparition de la cavitation et sa valeur théorique $\left|C_{p m}^{v i}\right|$, écart entre ses valeurs expérimentales trouvées sur modèle d'une part et sur prototype d'autre part, figures de cavitation qui dépendent du moyen d'essai utilisé [2] ou qui, plus encore, se modifient alors que les conditions globales qui déterminent l'écoulement: géométrie des

* Cette étude a été partiellement financée par la Direction des Recherches, Etudes et Techniques (convention 80144 ).

** Institut de Mécanique de Grenoble - BP 68 - 38402 , St Martin d'Hères Cedex. parois, vitesse de l'eau et pression ambiante, sont maintenues constantes.

Tous ces comportements, englobés parfois sous le terme d'effets d'échelle, manifestent que la recherche doit être attentive aux conditions de réalisation des écoulements et même aux procédures expérimentales. On doit suspecter a priori toutes les propriétés physiques de l'eau : tension superficielle, viscosité, propriétés thermiques, teneur globale en air dissous et teneur en germes libres. On doit prendre en compte aussi les caractéristiques de la turbulence en amont de la zone sensible à la cavitation.

En ce qui concerne le corps d'épreuve, il est nécessaire de considérer sa géométrie globale d'où résulte le gradient longitudinal de la pression, sa microgéométrie liée à la rugosité de la paroi et aussi les propriétés interfaciales solide-eau du matériau dont il est constitué. La procédure d'expérimentation elle-même peut être mise en cause notamment dans la manière de conduire les phases de transition d'un point de fonctionnement du tunnel hydrodynamique à un autre point en raison de la mémoire que l'eau garde des conditions antérieures par sa teneur en germes libres.

Dans l'histoire déjà longue des études concemant la cavitation, l'influence de chacun des points évoqués cidessus a été examinée plus ou moins profondément, comme le montre la lecture de l'ouvrage "Cavitation" de Knapp, Daily et Hammitt [3]. C'est cependant après l'année 1970 que la recherche s'est orientée d'une manière systématique vers l'étude de l'influence de la teneur de l'eau en germes libres d'une part (voir par exemple les références [4] à [6] pour une vue d'ensemble de cette question) et l'interaction entre l'état de la couche limite et la cavitation d'autre part [7] [8].

Deux faits saillants méritent d'être signalés ici. Dans le cas de cavitation attachée à une paroi, s'il existe un décollement laminaire de la couche limite, les figures de cavitation naissante s'établissent dans la zone de décollement. Dans le cas de cavitation développée à bulles 
séparées, la similitude des figures de cavitation exige que soit respecté, outre l'égalité des nombres $\sigma_{v}$ sur modèle et sur prototype, le rapport suivant des densités de germes libres : $N_{m} / N_{p} \simeq \lambda^{3}$, où $\lambda$ désigne le rapport des échelles de longueur [4]. Cette règle, d'origine purement géométrique, ne se réfère en rien à la dynamique des bulles et donc à leur taille et à leur pression critique. Elle suppose que les populations moyennes de germes sont telles que, dans chacun des écoulements, les conditions dynamiques permettent leur explosion. Sous cette réserve, elle donne une indication précieuse sur la nécessité d'effectuer les essais sur modèle réduit avec une eau beaucoup plus riche en germes que dans le cas du proto. type. Elle est donc de nature à limiter le champ d'application des règles de similitude que l'on peut déduire des études concernant l'interaction entre la cavitation et la couche limite : il se trouve en effet que nombre de ces études sont réalisées dans des conditions de très petite teneur en germes qui favorisent l'apparition de figures de cavitation dont il n'est pas sûr qu'elles existent encore à grande échelle. Cette restriction concerne aussi les résultats présentés ici, puisque l'expérimentation a été faite dans un tunnel hydrodynamique dont la concentration en germes était petite pour la plupart des essais effectués (inférieure à 0,3 par $\mathrm{cm}^{3}$ ), la pression critique des germes étant elle-même nettement inférieure à la pression de vapeur de l'eau.

Dans les cas de cavitation développée ${ }^{(1)}$ et de supercavitation qui nous concernent ici, on ne dispose pas de travaux ayant pour objet l'influence des effets visqueux ou de couche limite sur le détachement de la surface libre, frontière de la cavité, à partir d'une paroi solide à courbure continue. Nous nous sommes intéressés à cette question après que des essais ponctuels menés sur un cylindre circulaire [9] aient montré que la position du détachement de la cavité dépend fortement de là vitesse à nombre $\sigma_{v}$ constant. Les travaux antérieurs de notre équipe à l'Institut de Mécanique de Grenoble [10] qui concernent les ailes supercavitantes ou ventilées à arrière tronqué dont les points de détachement de la cavité sont déterminés a priori par une discontinuité de la tangente, méritaient alors de recevoir un prolongement dans cette direction. Le problème est double : qu'en est-il dans le cas où la tangente et la courbure de la paroi sont continues ? et, dans le cas des profils à arrière tronqué, les renseignements issus d'une étude sur la mutuelle influence de la cavitation et de la couche limite sont-ils susceptibles de donner quelque indication sur la stabilité du détachement au bord de fuite de l'extrados? Les deux questions ont un intérêt technique évident ; on remarquera en particulier que les efforts exercés sur une aile dépendent étroitement de la position des points de détachement des frontières de la cavité.

Par rapport aux travaux qui concernent la cavitation naissante, la situation envisagée présentement est plus complexe. En effet, s'il s'agit des conditions limites

(1) La frontière entre l'état de cavitation développée et celui de cavitation naissante ne peut être définie que d'une manière empirique. Nous ne pouvons utiliser ici la règle industrielle d'un pourcentage dans la chute des performances. Nous disons que la cavitation est développée lorsque les figures de cavitation sont permanentes et que leur taille est comparable à une dimension significative du corps. d'apparition de la cavitation, on admet aisément que la couche limite impose ses conditions, en particulier dans le cas d'un décollement laminaire qui offre un site favorable à la croissance des bulles séparées ou à la présence d'une très petite cavité attachée à la paroi. Au contraire, lorsque la cavitation est bien développée, les deux phénomènes coexistent au voisinage de la paroi et doivent s'accommoder l'un de l'autre : la cavitation ne peut plus être envisagée comme une petite perturbation de la couche limite établie antérieurement. Ce fait est illustré, en tunnel hydrodynamique, par la procédure d'essai qui consiste à abaisser la pression tout en maintenant constante la vitesse : la réduction de la pression ambiante, tout en faisant apparaître et développer la cavitation, modifie les gradients longitudinaux de pression et bouleverse donc la couche limite.

Nous présentons ici quelques résultats de deux recherches menées en parallèle, l'une sur un cylindre circulaire [11], l'autre sur deux profils elliptiques [12]. Ils concernent les aspects géométriques du détachement de la cavité, les efforts subis par le corps, l'état de la couche limite visualisée par l'injection de filet d'eau coloré à la fluorescéine, et enfin les indications données par un calcul de couche limite. On remarquera que ces résultats sont relatifs aux aspects globaux du phénomène : en l'absence d'études antérieures sur le sujet, il a paru nécessaire d'en acquérir d'abord une vue d'ensemble. Les profils elliptiques ont été choisis de manière à donner des gradients modérés de pression à incidence nulle et des pics de dépression pas trop profonds ni trop localisés près du bord d'attaque sous incidence, de manière à faire évoluer graduellement la couche limite. Cette double condition a fait choisir des profils dont les rapports d'axes étaient $1 / 4$ (profil 1) et $1 / 8$ respectivement (profil 2). Signalons enfin que, bien que les essais aient été effectués avec une très faible teneur de l'eau en germes, l'injection occasionnelle de quantités d'air assez importantes à l'amont de la section d'essais, d'où résultait l'explosion de grosses bulles au voisinage des profils, n'a pas modifié sensiblement les figures de cavitation attachées au corps : la question de la représentativité de nos résultats par rapport aux écoulements industriels à grande échelle reste donc ouverte.

\section{Moyen d'essais, similitude}

Les essais ont été menés dans la seconde veine à surface libre du Tunnel Hydrodynamique de l'Institut de Mécanique de Grenoble (longueur de la veine : $1600 \mathrm{~mm}$, largeur $120 \mathrm{~mm}$, hauteur d'eau : $400 \mathrm{~mm}$ ). La vitesse de l'eau peut y être fixée entre 2,5 et $12 \mathrm{~m} / \mathrm{s}$ environ et la pression à la surface libre peut y être abaissée jusqu'au voisinage de la tension de vapeur. Le taux de turbulence de l'écoulement est de $0,14 \%$ sur une largeur de $9 \mathrm{~cm}$ environ pour laquelle le profil des vitesses moyennes est uniforme ; il s'élève à $7 \%$ dans les couches limites qui se développent le long des parois verticales de la veine. Comme il a déjà été dit, aux pressions de fonctionnement du tunnel, la concentration en germes de cavitation dans l'eau est très petite.

Les corps d'épreuve sont les suivantes:

- un cylindre circulaire de diamètre $D=1 \mathrm{~cm}$ 
Po

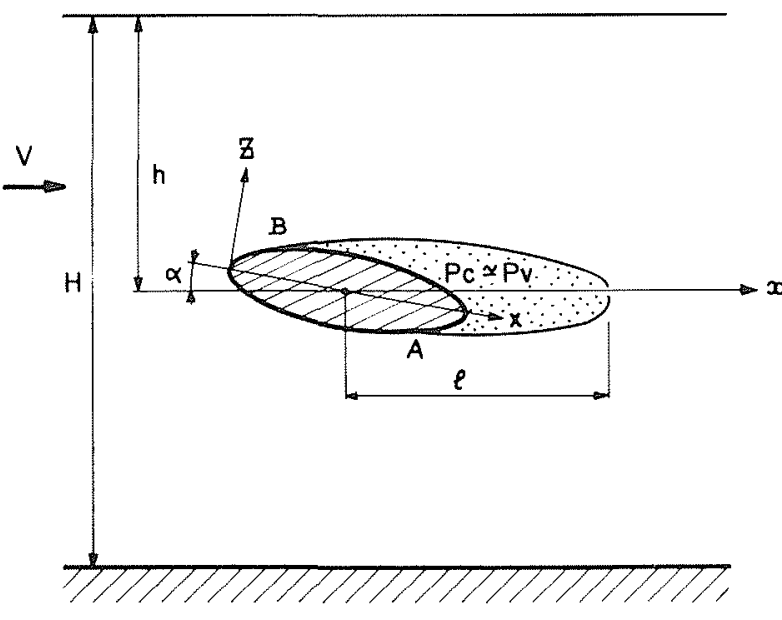

Figure 1-Schéma de l'écoulement.

- un cylindre elliptique de grand axe $2 a=8 \mathrm{~cm}$, de petit axe $2 b=2 \mathrm{~cm}$ (profil $\mathrm{n}^{\circ} 1$ )

- un cylindre elliptique de grand axe $2 a=8 \mathrm{~cm}$, de petit axe $2 b=1 \mathrm{~cm}$ (profil $\mathrm{n}^{\circ} 2$ ).

Ils sont placés de sorte que leur axe de symétrie soit à mi-hauteur du canal ( $h=H / 2=20 \mathrm{~cm}$, voir fig. 1).

Les paramètres de similitude que nous considérons sont les suivants :

- le nombre de cavitation $\sigma_{v}$ :

$$
\sigma_{v}=\frac{p_{0}+\rho g h-p_{v}}{\frac{1}{2} \rho V^{2}}
$$

où

$-p_{0}$.est la pression à la surface libre,

$-p_{v}$ la tension de vapeur de l'eau,

- V la vitesse moyenne à l'entrée.

En régime de cavitation développée, on suppose que la pression $p_{c}$ dans la cavité est peu différente de $p_{v}$, de sorte que la dépression relative de la cavité $\sigma$ est égale à $\sigma_{v}$.

- le nombre de Reynolds Re :

$$
\mathrm{Re}=\frac{\rho V L}{\mu}
$$

où $\mathrm{L}$ est une dimension significative du corps ( $D$ dans le cas du cylindre, $a$ dans le cas des profils elliptiques)

Dans ce dernier cas, il convient d'ajouter la variable incidence au nombre des paramètres adimensionnels indépendants.

Nous ne considérons pas les similitudes de Froude et de Weber, celle-ci devant prendre en compte d'ailleurs les tensions interfaciales eau-vapeur et eau-paroi solide. Pour la première, l'effet de gravité paraît a priori négligeable en raison des grandes valeurs du nombre de Froude et de la profondeur d'immersion $h$ comparée au diamètre $D$ du cylindre ou au petit axe $b$ des profils. Pour la seconde, nous l'éliminons provisoirement, attribuant à la similitude de Reynolds tous les effets de la vitesse de l'eau sur le détachement des cavités. Il s'agit là d'une hypothèse de travail qui se trouve partiellement justifiée par les résultats obtenus.

Les domaines de valeur des nombres $\sigma_{v}$ et $\operatorname{Re}$ sont montrés sur le tableau suivant:

\begin{tabular}{|c|c|c|c|c|}
\hline \multicolumn{3}{|c|}{ Tableau I } \\
\hline \multirow{2}{*}{ Corps } & \multicolumn{2}{|c|}{$\sigma_{v}$} & \multicolumn{2}{c|}{ Re } \\
\cline { 2 - 5 } & Mini & Maxi & Mini & Maxi \\
\hline Cylindre & 0,06 & 1,55 & 24000 & 106000 \\
\hline Profil 1 & 0,15 & 0,50 & 220000 & 545000 \\
\hline Profil 2 & 0,07 & 0,19 & 230000 & 380000 \\
\hline
\end{tabular}

L'incidence $\alpha$ est fixée entre -5 et +5 degrés.

\section{Géométrie du détachement}

\section{1. Cas du cylindre circulaire}

La figure 2 montre que les couches limites sur les parois latérales du canal n'affectent pas sensiblement la planéité de l'écoulement dans le voisinage du cylindre.



Figure 2. - Cylindre circulaire (vue générale) : $\sigma_{v}=0,701-$ $\mathrm{Re}=110000$.

Les figures 3 à 5 , avec mise au point sur l'extrémité du cylindre et profondeur de champ de l'ordre de $1 \mathrm{~cm}$, montrent quelques aspects du développement de la cavitation dans le sillage du cylindre. On peut y remarquer



Figure 3 - Cylindre circulaire $: \sigma_{v}=1,013-\operatorname{Re}=24600$. 


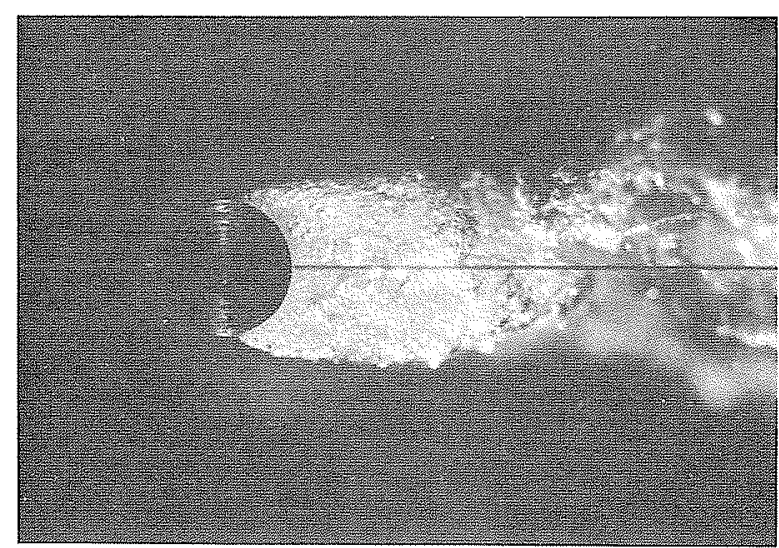

Figure 4 - Cylindre circulaire : $\sigma_{\boldsymbol{v}}=1,004-\mathrm{Re}=54900$.

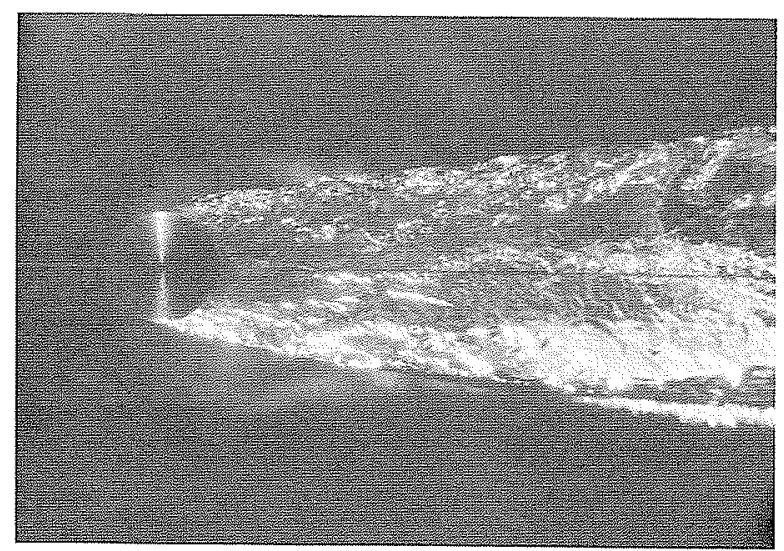

Figure 5 - Cylindre circulaire : $\sigma_{v}=0,196-\mathrm{Re}=54400$.

les variations de position et d'orientation, relativement au corps, du détachement de la cavité lorsque varient le nombre de Reynolds et le nombre de cavitation.

L'analyse systématique d'un grand nombre de telles photographies, correspondant à 92 points de fonctionnement du plan $\left(\mathrm{Re}, \sigma_{v}\right)$ - analyse comportant la digitalisation des frontières de la cavité sur table numérique et leur lissage - a permis d'obtenir, pour les angles $\varphi$ et $\psi$ caractérisant le détachement (Fig. 6) les valeurs moyennes $\bar{\varphi}$ el $\psi$ à partir de 28 réalisations. L'incertitude sur ces moyennes est de l'ordre de $\pm 1,4$ degré et

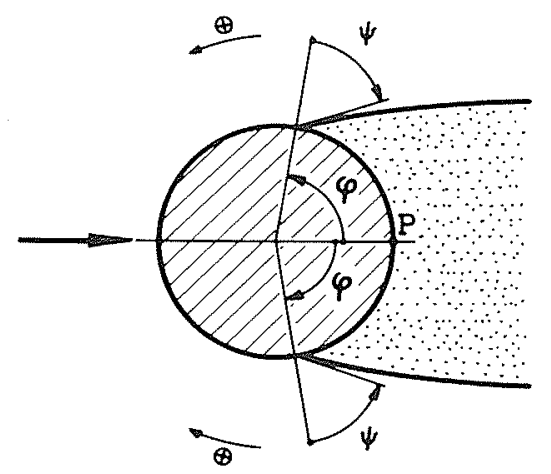

Figure 6 - Cylindre circulaire : repérage des points de détachement et de la direction du détachement. \pm 4 degrés respectivement. L'incertitude sur les angles $\varphi$ et $\psi$ eux-mêmes est de \pm 7 degrés, \pm 19 degrés respectivement ; elle doit être attribuée au caractère instationnaire du sillage de la cavité et donne une idée de l'amplitude des fluctuations des frontières de la cavité.

Les figures 7 et 8 donnent des exemples de variations de $\bar{\varphi}$ en fonction du nombre de Reynolds pour plusieurs valeurs de $\sigma_{v}$. A $\sigma_{v}$ constant - c'est-à-dire, grosso modo, à longueur de sillage constant - le point de détachement remonte vers l'amont lorsque le nombre de Reynolds augmente; il en est de même lorsque $\sigma_{v}$ diminue à vitesse constante, mais dans ce cas la longueur du sillage aug mente corrélativement. Les variations de $\bar{\varphi}$ et $\bar{\psi}$ en fonction de $\sigma_{v}$ sont montrées sur les figures 9 et 10 .

Une représentation analytique bi-linéaire par rapport à $\sigma_{v}$ et $1 / \operatorname{Re}$ de $\bar{\varphi}$ et $\bar{\psi}$ donne les résultats suivants :

$$
\begin{aligned}
& \bar{\varphi}=107,8-7,494 \sigma_{v}-\frac{915000}{\operatorname{Re}}-\frac{180000 \sigma_{v}}{\operatorname{Re}} \\
& \bar{\psi}=-88,32+10,93 \sigma_{v}+\frac{1860000}{\operatorname{Re}}-\frac{198300 \sigma_{v}}{\operatorname{Re}}
\end{aligned}
$$

pour le domaine de valeurs considéré de $\sigma_{v}$ et Re.

Elle correspond aux courbes en trait plein sur les figures 7 et 10 . On peut remarquer que l'extrapolation



Figure 7 - Cylindre circulaire : position du point de détachement en fonction du nombre de Reynolds - Influence de $\sigma_{v}$.

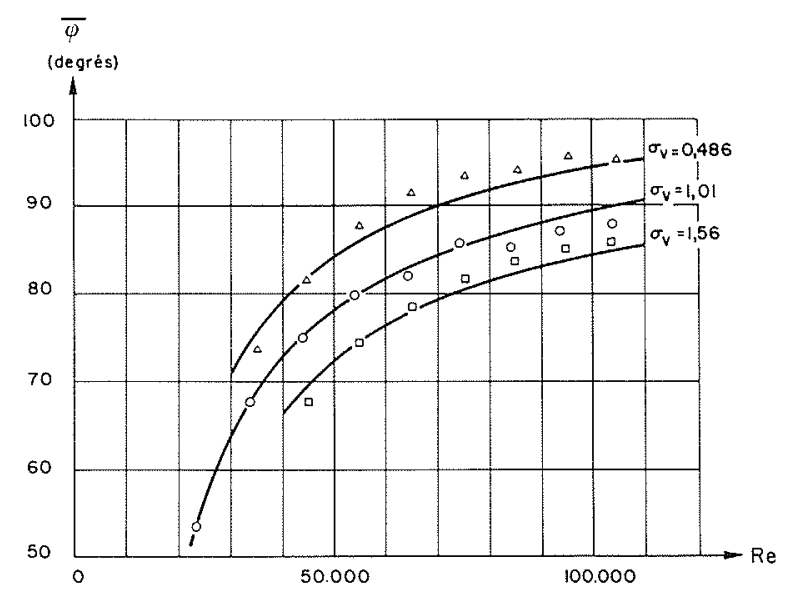

Figure 8 - Cylindre circulaire : position du point de détachement en fonction du nombre de Reynolds - Influence de $\sigma_{v}$. 


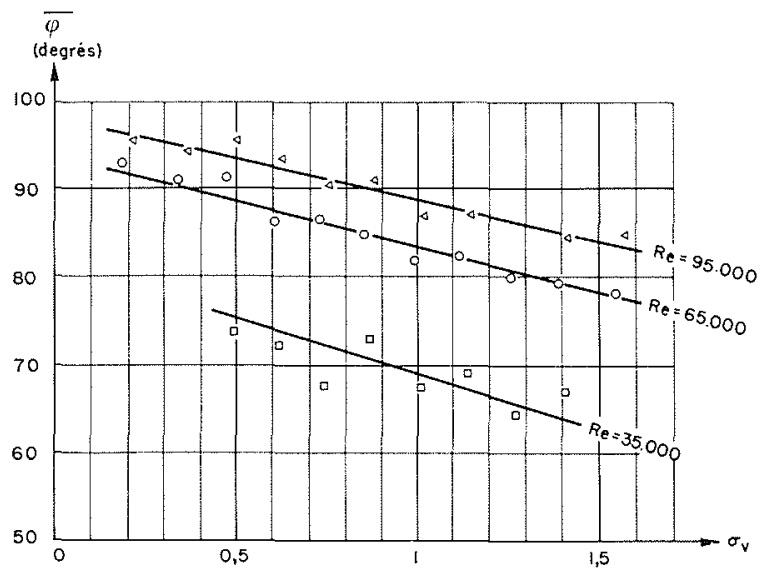

Figure 9 - Cylindre circulaire : position du point de détachement en fonction du paramètre de cavitation - Influence de Re.

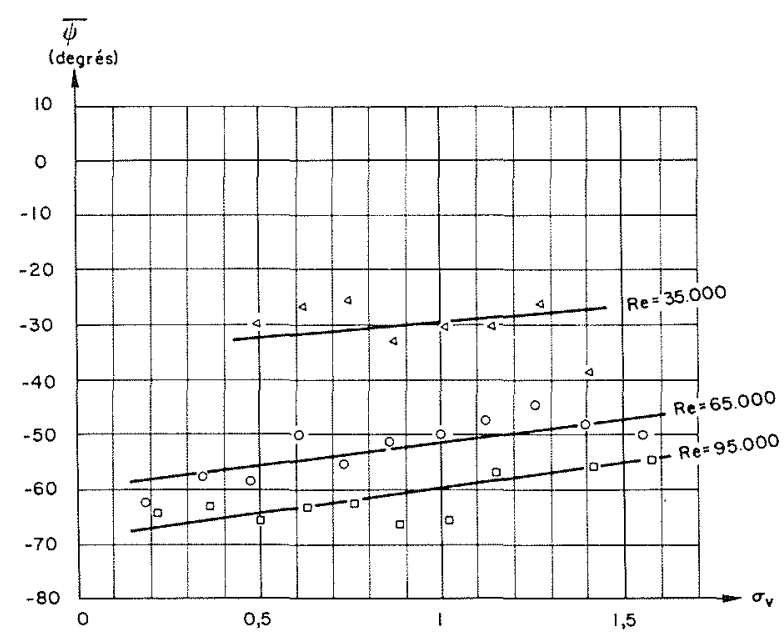

Figure 10 - Cylindre circulaire : direction du détachement en fonction de l'incidence $-\mathrm{Re}=$ constant - Influence de $\sigma_{v}$.

de la première relation au nombre de Reynolds $\mathrm{Re}=$ 190000 permet de trouver des valeurs de $\bar{\varphi}$ très proches de celles obtenues par Oba et al. [13]. Par contre, et inexplicablement, le sens de variation de $\bar{\psi}$ obtenu par ces auteurs est l'opposé de celui que nous constatons. Une autre extrapolation est tentante : $\sigma_{v}=1 / \mathrm{Re}=0$, qui correspond au cas du fluide non visqueux et de la cavité infinie ; la valeur de $\bar{\psi}$ montre que la ligne libre est alors à peu près tangente au cylindre ; la valeur de $\bar{\varphi}$, voisine de 108 degrés, est assez éloignée de celle que donne la théorie hydrodynamique pour un milieu infini, et qui est proche de 125 degrés.

Les résultats sur $\bar{\varphi}$ peuvent être comparés aussi aux prévisions semi-empliriques d'Arakeri [14] : si les tendances sont à peu près correctement rendues, il subsiste cependant une différence importante qui peut atteindre 20 degrés.

\subsection{Profil elliptique 1}

Les figures 11 et 12 montrent, pour deux valeurs de $\sigma_{v}$ et pour des incidences diverses, l'évolution de l'abscisse curviligne du point de détachement extrados $S_{d} / 2 a$ ( $S_{d}$ est comptée à partir du bord d'attaque) en fonction du nombre de Reynolds. L'influence du nom-

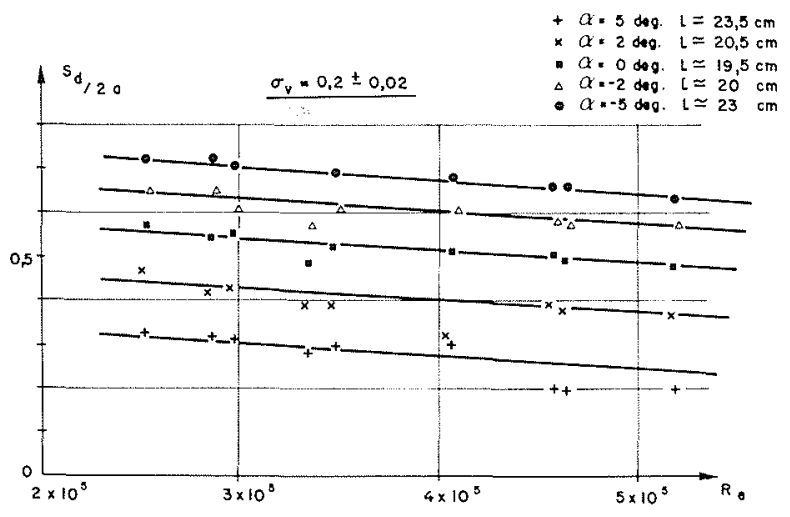

Figure 11 - Profil elliptique $n^{\circ} 1$ : position du point de détachement extrados en fonction du nombre de Reynolds - Influence de $\alpha$.

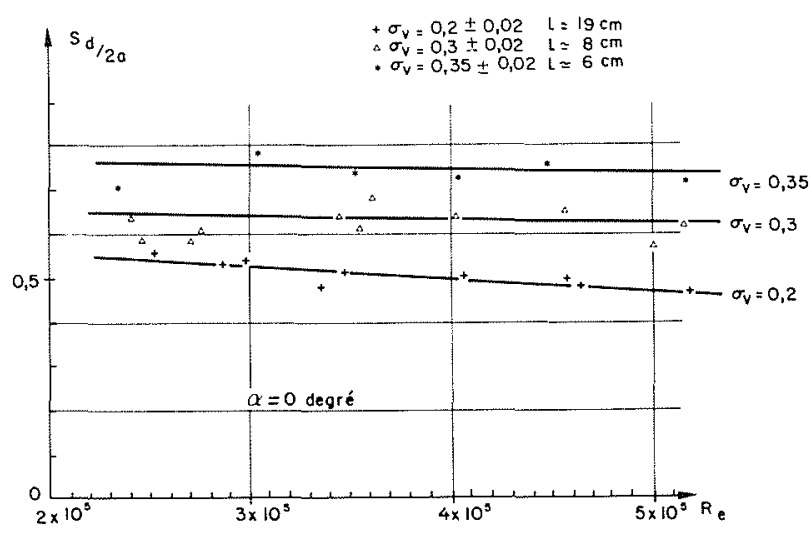

Figure 12 - Profil elliptique $\mathrm{n}^{0} 1$ : position du point de détachement en fonction du nombre de Reynolds - Influence de $\sigma_{v}$.

bre de Reynolds sur la position du point de détachement, bien que petite, est cependant nette. Elle est plus importante pour la plus petite valeur de $\sigma_{v}$ qui correspond au régime supercavitant. Ce fait surprenant ne peut être attribué à la définition de la dépression relative $\sigma$ de la cavité, prise ici égale à $\sigma_{v}$; on peut montrer en effet que, compte tenu des conditions de dégazage de notre tunnel, cette définition entraîne une erreur sur $\sigma$ au plus égale à $2 \%$.

On remarque, en général, que le détachement est plus stable lorsqu'il se produit dans les zones à forte courbure de l'ellipse, soit à l'amont, soit à l'aval.

\subsection{Profil elliptique 2}

De manière analogue, la figure 13 représente les variations de $S_{d} / 2 a$ en fonction de $\operatorname{Re}$ pour $\sigma_{v}$ constant. L'influence du nombre de Reynolds paraît ici peu importante. Aux incidences négatives et nulles, la position du point de détachement extrados est proche de l'arrière du profil et reste pratiquement fixée. Lorsque l'incidence varie de 2 à 4 degrés, le point de détachement passe assez brutalement de l'arrière à l'avant du profil. Dans l'intervalle, du moins pour les nombres $\sigma_{v}$ supérieurs à 0,12 environ, la cavitation disparaît presque complètement lorsque l'incidence est voisine de 3 degrés. Ce phénomène est visible sur les figures 21 


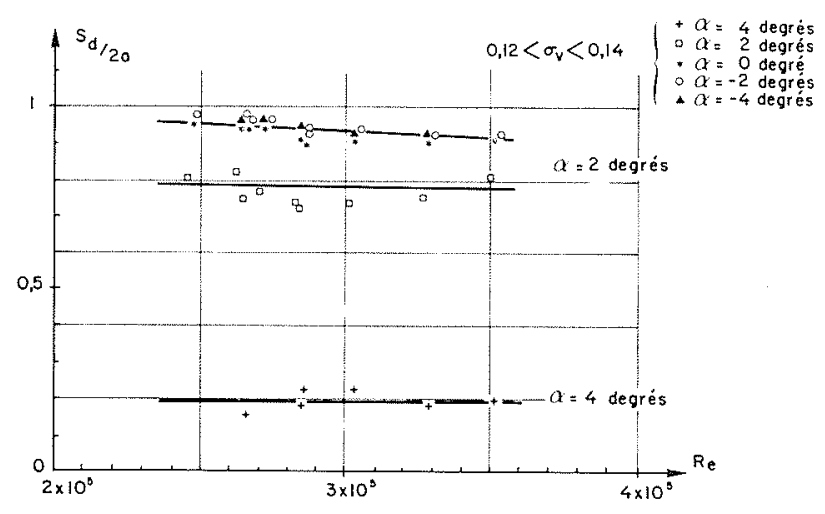

Figure 13 - Profil elliptique $n^{\circ} 2$ : position du point de détachement en fonction du nombre de Reynolds $-\sigma_{v}$ constant Influence de $\alpha$.

à 24. Il est observé aussi dans le cas du profil 1. Lorsque $\sigma_{v}$ est inférieur à 0,12 , la position du détachement varie de manière plus régulière : on observe un détachement stable, quoique très sensible aux petites variations des conditions expérimentales, sur la partie médiane de l'extrados.

\subsection{Conclusion sur la géométrie du détachement}

Les points principaux sont les suivants :

- L'influence du nombre de Reynolds sur le détachement de la cavité est plus marquée dans le cas du cylindre circulaire. Elle semble pratiquement inexistante pour le profil elliptique le plus mince ; cependant, la disparition de la cavitation sur les profils au voisinage de l'incidence 3 degrés laisse penser que les effets visqueux sont toujours présents.

- le détachement est plus stable lorsqu'il se produit sur les zones à forte courbure.

- l'écoulement autour du cylindre présente un caractère instationnaire plus marqué que les écoulements autour des profils elliptiques.

- la seule modélisation disponible ne rend pas compte correctement des phénomènes observés.

\section{Efforts}

Les efforts de portance et de traînée ont été mesurés dans le cas du profil 2 seulement, les dépassements de charge admissible sur la balance hydrodynamique n'autorisant pas la mesure dans le cas du profil 1 .

La figure 14 montre, en fonction de l'incidence et pour trois valeurs de $\sigma_{v}$, les variations des coefficients de portance et de traînée :

$$
C_{z}=\frac{Z}{\frac{1}{2} \rho V^{2} a e} \quad C_{x}=\frac{X}{\frac{1}{2} \rho V^{2} a e}
$$

où

- $e$ est la largeur de la veine,

- $X$ la traînée totale,

- Z la portance hydrodynamique, c'est-à-dire la portance mesurée diminuée de la poussée d'Archimède.
Sur la figure 14, chacun des points est indicé par la valeur des angles $\beta$ et $\beta^{\prime}$ par lesquels on exprime la position des points de détachement $\mathrm{B}$ et $\mathrm{A}$ à l'extrados et à l'intrados (Fig. 1).

L'interprétation des courbes obtenues est délicate puisque la géométrie de la partie mouillée du corps varie d'un point à l'autre. Cependant, pour les petites valeurs de l'incidence, les variations $C_{z}(\alpha)$ sont pratiquement linéaires. La pente des droites varie surtout en raison $\mathrm{du}$ plus ou moins grand dénoiement du corps lié à la valeur de $\sigma_{v}$.

Un fait mérite d'être souligné : toutes les droites $C_{z}(\alpha)$ passent par le point de portance nulle à une incidence voisine de -1 degré. On remarque en même temps que les points de détachement à l'extrados et à l'intrados sont symétriques. Il est intéressant alors de rattacher ce fait à la notion d'incidence d'adaptation. On sait que cette notion exprime le moindre contournement du bord d'attaque par le fluide, ce qui se traduit, en théorie linéarisée, par la disparition d'un pôle sur la vitesse de perturbation. L'expérience acquise antérieurement a permis de vérifier que dans de nombreux cas la portance théorique à l'incidence d'adaptation $C_{z}\left(\alpha_{a}\right)$ dépend très peu des conditions extérieures : longueur de cavité, profondeur d'immersion, distance au fond du canal ... En même temps, il se trouve que la portance expérimentale, pour la valeur moyenne $\alpha_{m}$ du domaine d'incidences exemptes de cavitation au bord d'attaque, est elle-même constante et égale à $C_{z}\left(\alpha_{a}\right)$. On peut donc penser que la portance $C_{z}\left(\alpha_{a}\right)$ exprime une qualité propre au profil. Dans le cas du profil elliptique, la symétrie implique que $C_{z}\left(\alpha_{a}\right)$ soit nul. L'expérience semble montrer alors que ceci est réalisé par une distribution symétrique des pressions et donc des gradients de pression à l'extrados et à l'intrados, de sorte que les couches limites et les points de détachement sont euxmêmes symétriques. Les profils symétriques sont donc particulièrement favorables à l'étude de l'interaction entre la cavitation développée et la couche limite.

La figure 15 montre, à $\sigma_{v}$ constant, l'influence du nombre de Reynolds sur la courbe $C_{z}(\alpha)$. Cette influence est considérable : à l'incidence 1 degré, par exemple, $C_{z}$ augmente de $50 \%$ lorsque la valeur de Re passe de $3,53 \times 10^{5}$ à $2,33 \times 10^{5}$, alors même que la géométrie de la partie mouillée ne varie pas beaucoup. On voit aussi que le décrochement par passage à la supercavitation à extrados dénoyé se fait pour des valeurs de $\alpha$ et $C_{z}$ qui dépendent fortement du nombre de Reynolds. L'influence du nombre de Reynolds est représentée d'une autre manière sur la figure 16: elle disparaît pratiquement à l'incidence - 1 degré, voisine de l'incidence d'adaptation, et devient plus marquée lorsqu'on s'éloigne de cette incidence. On voit aussi qu'elle augmente quand le nombre de cavitation augmente.

En résumé, on voit que le coefficient de portance dépend fortement du nombre de cavitation et du nombre de Reynolds. La première dépendance était attendue puisque la géométrie de la partie mouillée est évidemment en relation avec le développement de la cavitation. La seconde dépendance, par contre, pose un problème puisqu'il a été vu que le nombre de Reynolds n'influe pratiquement pas sur la position des 


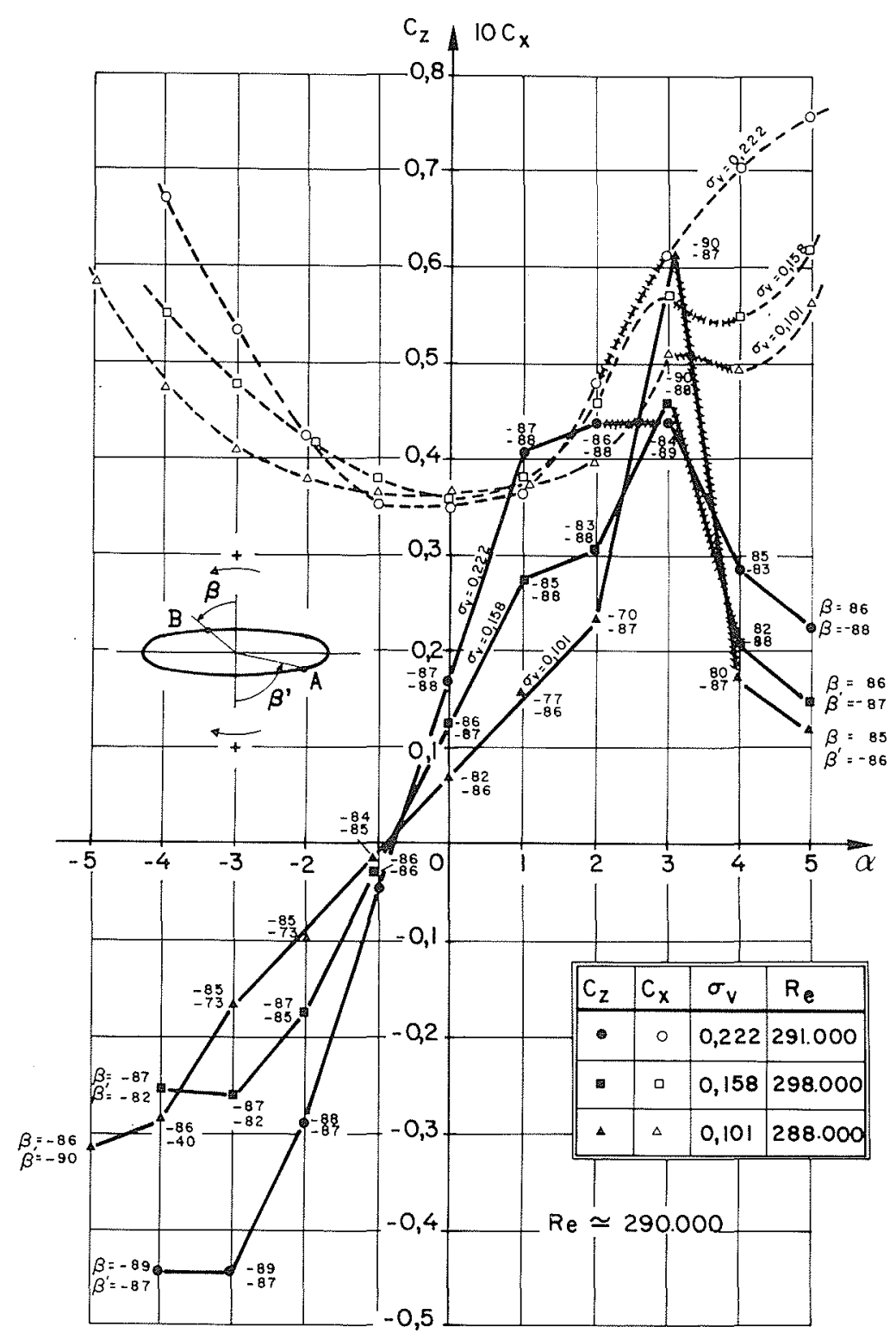

Figure 14 - Profil elliptique $n^{\circ} 2$ : coefficients de portance et de traînée en fonction du nombre de Reynolds $=$ Influence de $\sigma_{v}$ et de $\alpha$.

points de détachement dans le cas du profil elliptique mince que nous considérons ici. Quel est le mécanisme sous-jacent à cette dépendance ? Il n'est pas possible, à l'heure actuelle, de répondre à cette question.

\section{Couche limité}

\subsection{Visualisation}

La visualisation de la couche limite a été effectuée par l'injection d'un filet d'eau très mince (diamètre $0,2 \mathrm{~mm}$ ), coloré à la fluorescéine, en un point situé au sommet amont du grand axe du profil elliptique $\mathrm{n}^{\circ} 2$, c'est-à-dire dans une zone où la surpression dynamique due au point d'arrêt compense le risque de cavitation qui tendrait à s'accrocher à l'orifice d'injection. L'expérience montre qu'on obtient ainsi une bonne idée de l'état global de la couche limite, et particulièrement de la position du point de décollement laminaire et du point de transition à la turbulence.

Les figures 17 à 20 montrent, dans le cas subcavitant, l'état de la couche limite à des incidences de 5, 3,2 et 0 degrés respectivement, pour un nombre de Reynolds de $2,58 \times 10^{5}$, c'est-à-dire pour une vitesse de l'eau de l'ordre de $6,5 \mathrm{~m} / \mathrm{s}$. A 5 degrés, on distingue un décollement local suivi d'un réattachement et d'une transition. A 4 degrés (non montré ici) le décollement laminaire disparaît, la transition se fait à une abscisse relative de $6,4 \%$. A 3 degrés (Fig. 18) la transition évolue vers l'aval à une abscisse de $21 \%$. A 2 degrés, le changement d'aspect de la couche limite est important : elle se développe jusqu'à la partie aval de l'extrados : la figure 19 fait apparaître un décollement laminaire suivi d'un réattachement et d'une transition entre les abscisses relatives 90 et $98 \%$. A 1 degré et 0 degré (Fig. 20), on a très nettement un décollement laminaire suivi d'enrou- 


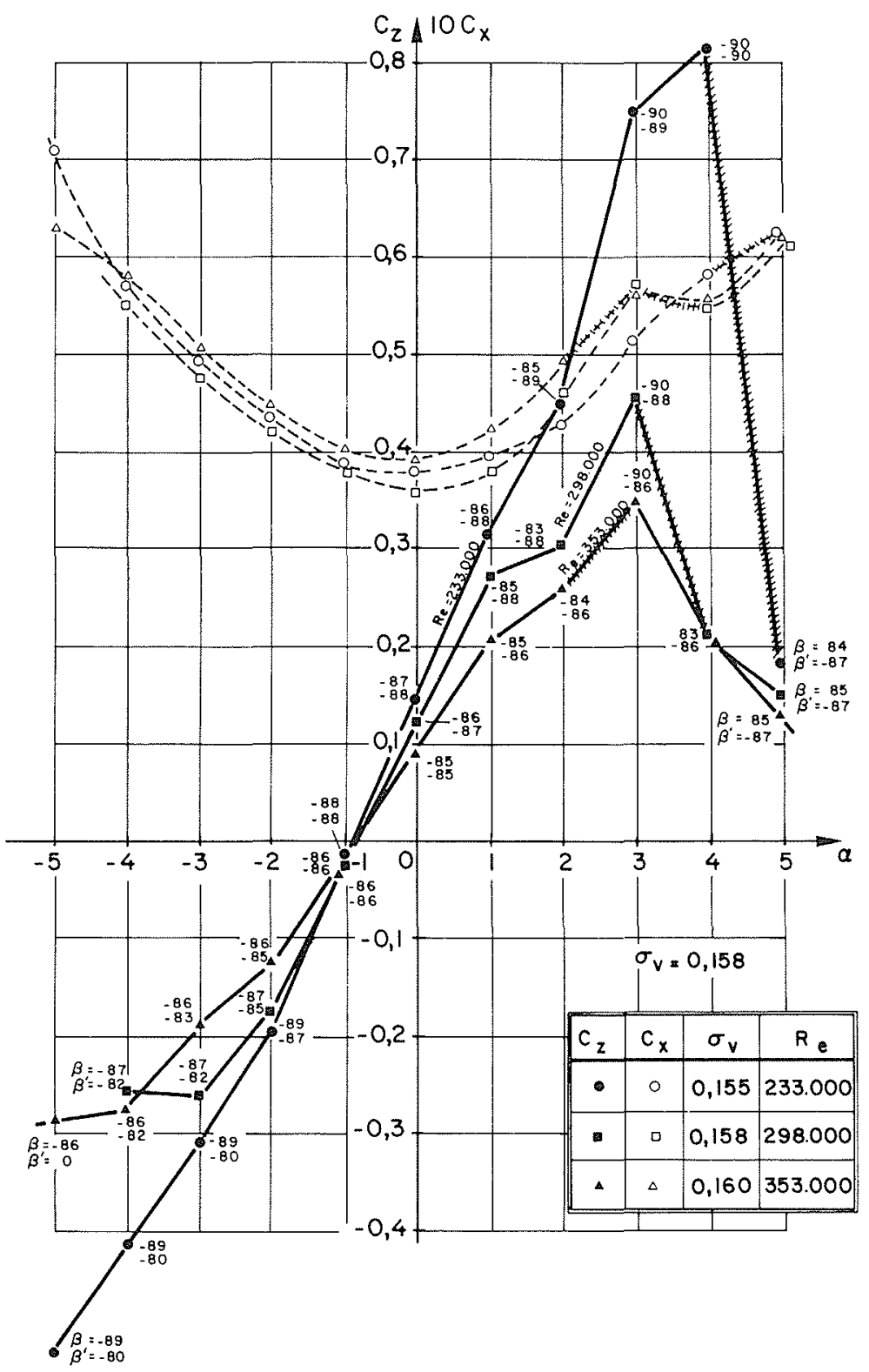

Figure 15 - Profil elliptique $\mathrm{n}^{\circ} 2$ : coefficients de portance et de traînée en fonction de l'incidence $-\sigma_{v}$ constant - Influence de Re.

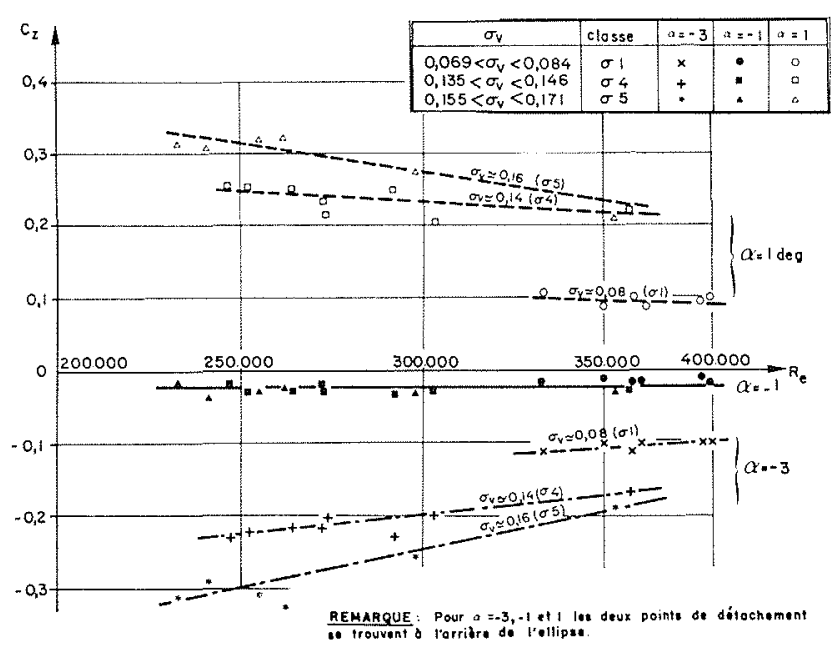

Figure 16 - Profil elliptique $\mathrm{n}^{\circ} 2$ : coefficients de portance en fonction du nombre de Reynolds - Influence de $\sigma_{v}$ et de $\alpha$. lements d'instabilité (abscisses relatives $91 \%$ et $86 \%$ à 1 et 0 degré respectivement).

Les figures 21 à 24 sont relatives au cas de cavitation développée $\left(\sigma_{n}=0,147, \operatorname{Re}=3,22 \times 10^{6}\right)$. Pour les incidences 5 degrés et 4 degrés, la cavitation se détache assez près du bord d'attaque. A 4 degrés par exemple (Fig. 21), le filet coloré contoume la zone de cavitation avec un décollement presque tangentiel de type laminaire ; il est dévié du plan vertical d'origine par les aspects tridimensionnels locaux dûs à la formation de "doigts" limités en envergure. A l'incidence 3 degrés, il est tout à fait remarquable que la cavitation disparaisse à peu près complètement, avec un point de transition situé à une abscisse relative de $35 \%$ environ (Fig. 22), alors qu'elle réapparaît nettement aux incidences de 2 et 0 degré (Fig. 23 et 24) avec un détachement précédé d'un décollement laminaire. Le même phénomène a été constaté à plusieurs nombres de Reynolds. Il semble bien que la disparition de la cavitation doive être liée 


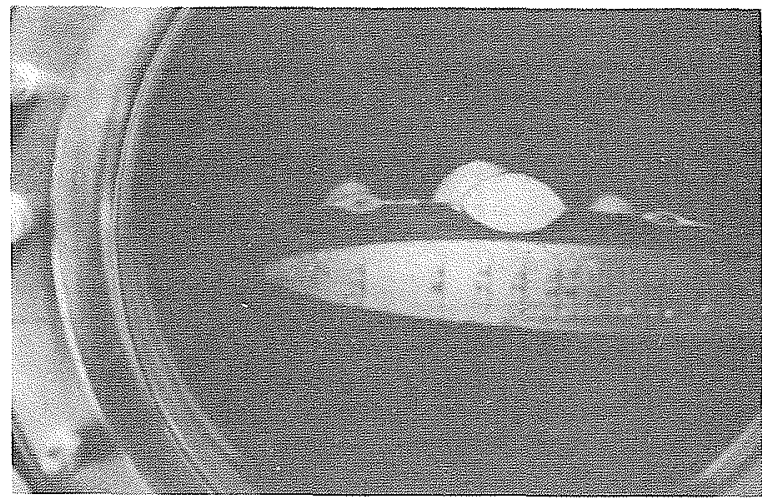

Figure 17 - Profils elliptique $n^{\circ} 2$. Régime subcavitant $\mathrm{Re}=258000-\alpha=5$ degrés.

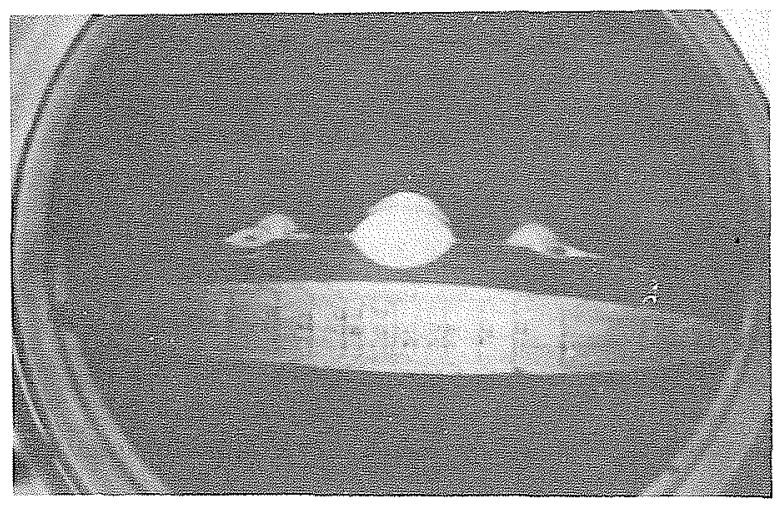

Figure 18 - Profil elliptique $n^{0} 2$ - Régime subcavitant $\operatorname{Re}=258000-\alpha=3$ degrés.

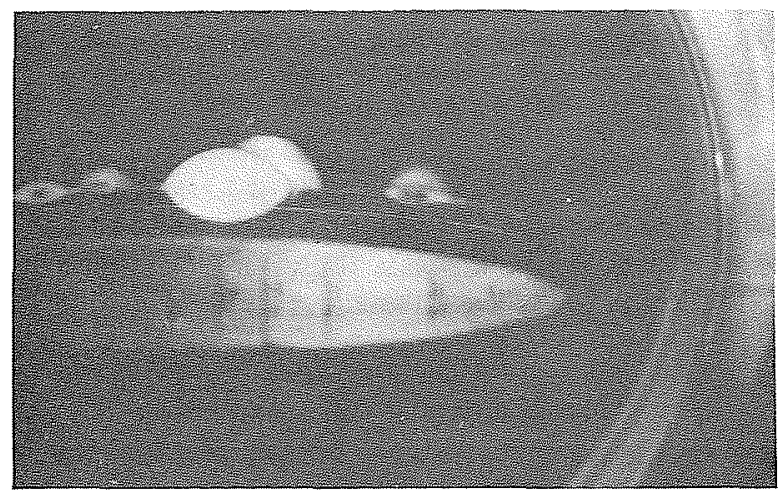

Figure 19 - Profil elliptique $\mathrm{n}^{\circ} 2$ - Régime subcavitant $\operatorname{Re}=258000-\alpha=2$ degrés.

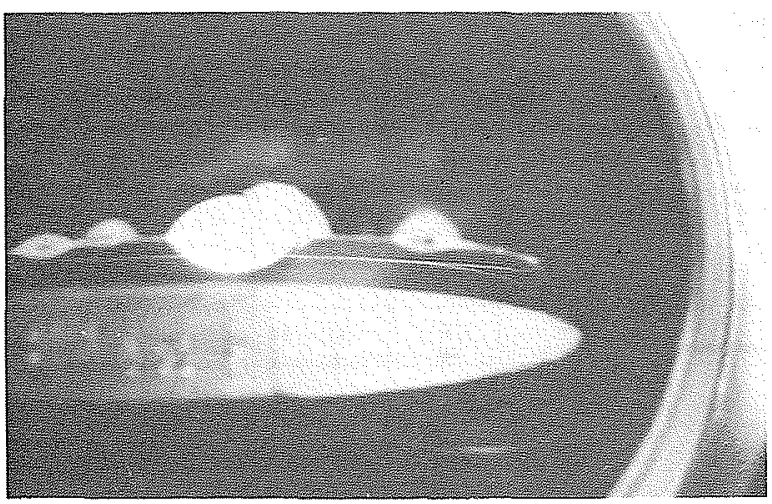

Figure 20 - Profil elliptique $n^{\circ} 2-$ Régime subcavitant $-\mathrm{Re}=$ $258000-\alpha=0$ degré.

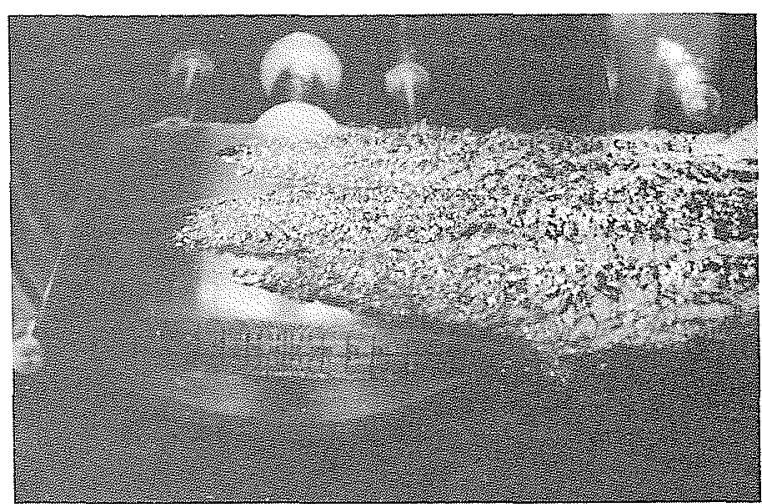

Figure $21-$ Profil elliptique $n^{\circ} 2-\sigma_{v}=0,147-\operatorname{Re}=322000$ $-\alpha=4$ degrés.

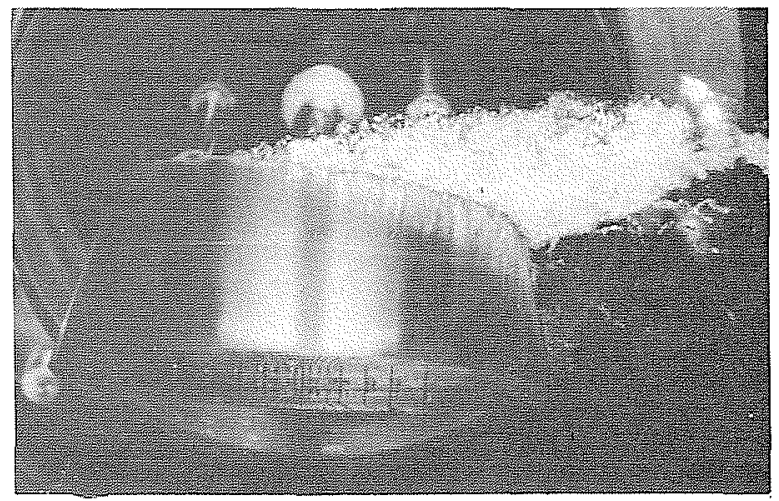

Figure $22-$ Profil elliptique $\mathrm{n}^{0} 2-\sigma_{v}=0,147-\mathrm{Re}=322000$ $-\alpha=3$ degrés.

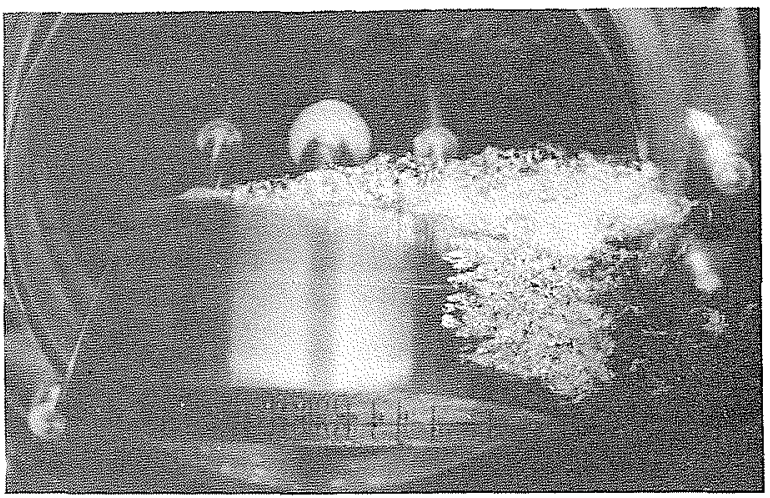

Figure $23-$ Profil elliptique $\mathrm{n}^{\circ} 2-\sigma_{v}=0,147-\mathrm{Re}=322000$ $-\alpha=2$ degrés.

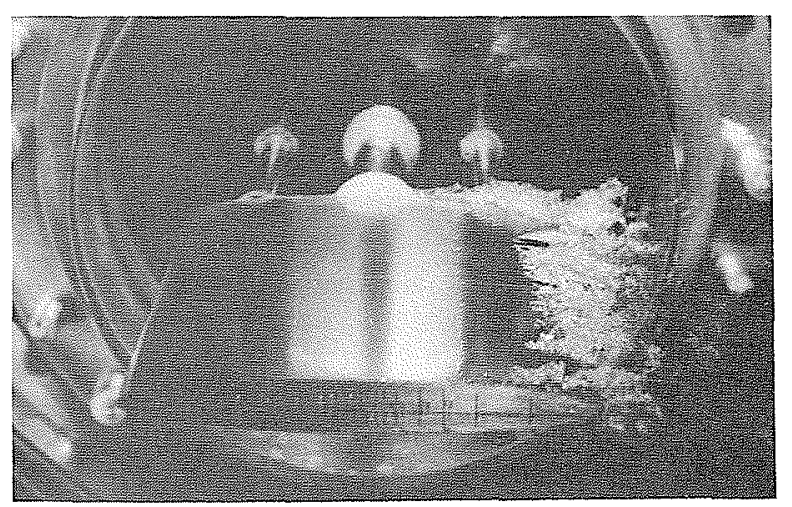

Figure 24 - Profil elliptique $\mathrm{n}^{\circ} 2-\sigma_{v}=0,147-\mathrm{Re}=322000$ $-\alpha=0$ degré. 
Tableau II

\begin{tabular}{|c|c|c|c|c|c|c|c|c|c|c|}
\hline \multirow[b]{2}{*}{ Numéro } & \multirow{2}{*}{$\begin{array}{c}\alpha \\
\text { expérience } \\
\text { (degrés) }\end{array}$} & \multirow{2}{*}{$\begin{array}{c}V \\
(\mathrm{~m} / \mathrm{s})\end{array}$} & \multirow[b]{2}{*}{$\operatorname{Re}$} & \multirow[b]{2}{*}{$\sigma_{v}$} & \multirow{2}{*}{$\begin{array}{c}\alpha \\
\text { ajusté } \\
\text { (degrés) }\end{array}$} & \multicolumn{2}{|c|}{$\ell(\mathrm{cm})$} & \multirow{2}{*}{$c_{z}$} & \multicolumn{2}{|c|}{$C_{x}$} \\
\hline & & & & & & expérience & ajusté & & expérience & théorie \\
\hline 1 & 0 & 10,15 & 379000 & 0,0686 & 1,85 & 13 & 7,0 & 0,0429 & 0,0168 & 0,0154 \\
\hline 2 & 0 & 9,95 & 361000 & 0,0749 & 1,10 & 14 & 9,2 & 0,0389 & 0,0172 & 0,0152 \\
\hline 3 & 0 & 9,07 & 333000 & 0,0833 & 0,91 & 11 & 7,8 & 0,0451 & 0,0173 & 0,0168 \\
\hline 4 & 0 & 9,03 & 323000 & 0,0989 & 0,91 & 9,2 & 6,3 & 0,0370 & 0,0195 & 0,0204 \\
\hline 5 & 0 & 9,46 & 346000 & 0,107 & 1,32 & 7,0 & 5,1 & 0,0514 & 0,0236 & 0,0243 \\
\hline 6 & 0 & 8,74 & 331000 & 0,114 & 1,25 & 4,5 & 4,0 & 0,0978 & 0,0230 & 0,0328 \\
\hline 7 & 0 & 8,41 & 303000 & 0,137 & 0,91 & 5,1 & 4,0 & 0,104 & 0,0237 & 0,0356 \\
\hline 8 & 2 & 8,74 & 331000 & 0,114 & 2,52 & 5,0 & 4,1 & 0,369 & 0,0305 & 0,0339 \\
\hline 9 & 2 & 8,41 & 303000 & 0,137 & 2,98 & 5,5 & 4,5 & 0,293 & 0,0304 & 0,0343 \\
\hline
\end{tabular}

au développement de la turbulence dans la couche limite : des faits semblables avaient déjà été observés dans le cas d'une transition à la turbulence provoquée artificiellement par un fil tendu sur le nez d'un corps de révolution [15].

\subsection{Calcul de la couche limite}

L'ambition de ce calcul est de corréler si possible les caractères globaux de la couche limite qu'on en déduit, notamment la position du décollement laminaire et de la transition à la turbulence, avec les observations expérimentales.

Il se déroule pratiquement en deux étapes :

- d'une part, on calcule dans un schéma purement hydrodynamique les coefficients de pression ainsi que les efforts globaux, portance et traînée;

- d'autre part, on effectue le calcul proprement dit de couche limite, en utilisant comme donnée d'entrée la répartition de coefficients de pression trouvée dans la première étape.

La première étape utilise le modèle non linéaire de Pellone [16] basé sur la méthode des singularités discrétisées et relatif à des écoulements supercavitants bi ou tri-dimensionnels autour d'ailes à extrados noyé placées sous une surface libre. Les données d'entrée sont, outre la géométrie du profil, son incidence et sa profondeur d'immersion, la longueur de la cavité et la position des points de détachement d'intrados et d'extrados A et $B$ (Fig. 1). Un fait constant de ce genre de modèle est de donner une valeur trop petite à la dépression relative de la cavité $\sigma$. De même, à une incidence donnée, le coefficient de portance théorique diffère souvent de la valeur expérimentale par suite de la grande valeur de la pente $\partial C_{z} / \partial \alpha$. On ajuste alors par tâtonnement la longueur de la cavité et l'incidence pour obtenir les valeurs expérimentales de $\sigma$ et de $C_{z}$ et c'est la répartition correspondante des coefficients de pression qui est utilisée dans le programme de calcul de la couche limite. Dans la plupart des cas, le rapport de la longueur corrigée à la longueur expérimentale de la cavité est voisin de 0,7 tandis que l'écart des incidences est de l'ordre de un degré.
Le programme de calcul de couche limite a été développé à l'ONERA-CERT par Arnal et Habiballah [17]. Il utilise la méthode intégrale et donne, outre les épaisseurs de déplacement, de quantité de mouvement et d'énergie cinétique, les abscisses des points de décollement laminaire, de début de transition, éventuellement de recollement et de régime de turbulence développée. Il est mené à partir du point d'arrêt jusqu'au point de détachement $\mathrm{B}$ de la cavité à l'extrados du profil.

Les tableaux II et III sont relatifs à des résultats obtenus très récemment et ayant de ce fait un caractère encore incomplet. Le tableau II présente les caractéristiques des points expérimentaux étudiés ainsi que les résultats du calcul de Pellone, dans les cas des incidences expérimentales 0 et 2 degrés. On y montre aussi les valeurs ajustées de l'incidence et de la longueur de cavité. On remarque en particulier que, sauf pour les points numérotés 6 et 7, l'accord entre théorie et expérience, en ce qui concerne la traînée, peut être considéré comme bon puisque l'erreur relative est de l'ordre de ou inférieure à $10 \%$.

En ce qui concerne le calcul de la couche limite, le tableau $n^{\circ}$ III donne un résultat remarquable dans le cas des points numérotés 2 à 7 puisque, conformément

Tableau III

\begin{tabular}{|c|c|c|c|c|c|}
\hline Numéro & $\begin{array}{c}S_{d} \\
(\mathrm{~mm}) \\
\text { expérience }\end{array}$ & $\begin{array}{c}S_{D L} \\
(\mathrm{~mm}) \\
\text { calcul }\end{array}$ & $\frac{S_{d}-S_{D L}}{2 \mathrm{a}}$ & $\begin{array}{c}S_{D T} \\
(\mathrm{~mm}) \\
\text { calcul }\end{array}$ & $\frac{S_{d}-S_{D T}}{2 \mathrm{a}}$ \\
\hline 1 & 58 & - & - & 52,7 & 0,066 \\
\hline 2 & 55 & 51,9 & 0,044 & 53,8 & 0,020 \\
\hline 3 & 58 & 54,7 & 0,040 & 57,5 & 0,005 \\
\hline 4 & 59 & 56,8 & 0,027 & 58,6 & 0,004 \\
\hline 5 & 61 & 57,8 & 0,044 & 60,6 & 0,009 \\
\hline 6 & 72 & 67,7 & 0,050 & 68,3 & 0,043 \\
\hline 7 & 72 & 71,7 & 0,006 & 71,8 & 0,004 \\
\hline 8 & 70 & - & - & 56,0 & 0,174 \\
\hline 9 & 59 & - & - & 48,0 & 0,141 \\
\hline
\end{tabular}


à l'expérience, le calcul fait apparaître un décollement laminaire un peu en amont du détachement de la cavité (sur ce tableau, $S_{d}$ désigne l'abscisse curviligne du point de détachement $\mathrm{B}, S_{D L}$ et $S_{D T}$ sont relatives au point de décollement laminaire et au début de transition respectivement). Ce résultat doit être encore soumis à des vérifications : on doit tester, en particulier, qu'aucune irrégularité numérique dans le calcul des coefficients de pression n'engendre un décollement laminaire ou un début de transition fictifs. S'il est confirmé, il donne une indication précieuse sur l'importance du décollement laminaire et, plus généralement, sur l'influence prépondérante des effets visqueux par rapport au détachement de la cavité.

Il est utile aussi de considérer les résultats correspondant aux points 1, 8 et 9 des tableaux II et III. En ce qui concerne le point numéro 1 , on remarque l'absence de décollement laminaire ; cependant l'examen de l'évolution des paramètres de forme montre que dans une situation très voisine le début de transition et le décollement laminaire seraient pratiquement confondus ; on remarque aussi que l'écart entre l'incidence ajustée et l'incidence expérimentale est assez grand dans le cas du point numéro 1. Dans les cas des points 8 et 9 , la transition à la turbulence apparaît seule, et à une distance du point de détachement $B$ plus grande que dans les cas précédents puisqu'elle est supérieure au centimètre au lieu de l'ordre du millimètre. Ce fait peu être rapproché des observations expérimentales concernant la transition à la turbulence pour les incidences voisines de 3 degrés, transition qui fait disparaître les figures de cavitation.

\section{Conclusion}

Les résultats présentés ici mettent en évidence une forte interaction entre le détachement de la frontière d'une cavité à partir d'une paroi solide à courbure continue et le développement de la couche limite sur cette paroi. En particulier, les résultats expérimentaux relatifs aux profils elliptiques confirment le lien qui existe entre le décollement laminaire de la couche limite et le détachement de la frontière de la cavité, même dans le cas de supercavitation; ils confirment aussi le rôle négatif de la couche limite turbulente par rapport à la cavitation attachée à une paroi, au moins dans le cas de nombres de cavitation modérés. Les résultats relatifs au cylindre circulaire montrent que la modélisation semi-empirique d'Arakeri, tout en donnant les bonnes tendances sur la position du détachement, comporte cependant un défaut de calage assez important. Nous avons alors utilisé une procédure d'analyse théorique analogue à celle qui est pratiquée couramment en Aéronautique : calcul des coefficients de pression d'abord, puis calcul de la couche limite. Cette procédure souffre dans notre cas de handicaps dont le plus important est la nécessité d'une information préalable sur la position du détachement de la cavité. Les premiers résultats indiquent cependant que les caractères principaux de la couche limite concernant le décollement laminaire et la transition à la turbulence sont décrits avec une certaine précision par le modèle théorique. Il reste à la développer suffisamment pour qu'il soit possible d'en tirer des règles de prédiction sur les figures de cavitation développée et sur la stabilité du détachement.

\section{Références}

11] VILLAT H. - Sur la validité des solutions de certains problèmes d'hydrodynamique. J. Math. Pures et Appliquées 6, 10 (1914).

[2] LINDGREN H., JOHNSSON C.A. - Cavitation inception on head forms, ITTC comparative experiments. Proceedings 11 th International Towing Tank Conference, Tokyo 1966, pp. $219-232$.

[3] KNAPP R.T., DAILY J.W., HAMMITT F.G. - Cavitation. Mc Graw Hill ed., 1970.

(4) LECOFFRE Y., MARCOZ J., VALIBOUSE B. - Aspects pratiques du contrôle des germes de cavitation en moyen d'essais. $11^{\mathrm{e}}$ Symposium de la Section "Hydraulic Machinery, Equipment and Cavitation" de l'A.I.R.H., Amsterdam, 13-17 Septembre 1982.

[5] OLDENZIEL D.L., JANSEN R.H.J., KELLER A.P. LECOFFRE Y., VAN RENSSE R.L. - Comparison of instruments for the detection of bubbles and particles in water during cavitation studies. $11^{\mathrm{e}}$ Symposium de la Section "Hydraulic Machinery, Equipment and Cavitation" de l'A.I.R.H., Amsterdam, 13-17 Sep tembre 1982.

[6] OLDENZIEL D.L. - Utility of available instruments during cavitation tests. 11 e Symposium de la Section "Hydraulic Machinery, Equipment and Cavitation" de l'A.I.R.H., Amsterdam, 13-17 Septembre 1982.

[7] ARAKERI V.H., ACOSTA A. - Viscous effects in the inception of cavitation. International Symposium on Cavitation Inception, A.S.M.E., New-York (1979).

[8] VAN DER MEULEN J.H.J. - Boundary layer and cavitation studies of NACA 16.012 and NACA 4412 Hydrofoils. 13 th'. Symposium on Naval Hydrodynamics, Tokyo, Octobre 1980, pp. 195-219.

[9] MICHEL J.M. - Detachment of free surface from solid walls with continuous curvature in supercavitation flows: some observations. Colloque Euromech 116 "Laminar separation and transition, and their possible connection with cavitation", Wageningen, 28-30 Mai 1979.

[10] DODU J., MICHEL J.M., ROWE A. - Les écoulements autour des ailes ventilées. La Houille Blanche, $n^{\circ}$ 5-6, 1976, pp. 449-457.

[11] FRANC J.P. - Etudes de cavitation. II. Sillages cavitants d'obstacles épais. Etude physique. Thèse de Docteur-Ingénieur, Grenoble, 30 Septembre 1982.

[12] BISET S. - Cavitation développée sur une paroi à courbure continue. Etude physique du détachement. Thèse de Docteur-Ingénieur, Grenoble, 25 Juin 1982.

[13] OBA R., IKOHAGI T., YASU S. - Supercavitating cavity observations by means of laser velocimeter. J. of Fluids Engineering, Dec. 1980, vol. 102, pp. 433-438.

[14] ARAKERI V.H. - Viscous effects on the position of cavitation separation from smooth bodies. $J$. of Fluids Mechanics, 1975, vol. 68, part 4, pp. 779-799.

[15] ARAKERI V.H., ACOSTA A.J. - Cavitation inception observations on axisymmetric bodies at supercritical Reynolds numbers. $J$. of Ship Research, vol, $20, \mathrm{n}^{\circ} 1,1976$, pp. 40-50.

[16] PELLONE C., ROWE A. - Supercavitating hydrofoils in non linear study. $3^{\mathrm{e}}$ Conf. Internationale d'Hydrodynamique Navale Numérique, O.N.R.-D.G.A., Paris, Juin 1981.

117] ARNAL D., HABIBALLAH M. DELCOURT V. - Syn thèse sur les méthodes de calcul de la transition développée au D.E.R.A.T. O.N.E.R.A.-C.E.R.T.-D.E.R.T., rapport technique $\mathrm{n}^{\circ} 11 / 5018$ AYD, Juin 1980. 
Discussion

Président: S. CASACCI

Après avoir félicité M. MICHEL, M. BONNIN s'étonne de ce que contrairement aux coefficients de portance et de trainée le coefficient de moment n'ait pas été analysé. M. MICHEL explique ce fait par les difficultés de mesure du moment.

M. MICHEL précise ensuite que l'interaction décollementcavité dépend surtout des valeurs des nombres de REYNOLDS et de $\sigma$. Il ajoute que la méthode présentée est une méthode d'analyse nécessitant la connaissance préalable des points de détachement et ne permettant donc pas, à theure actuelle, d'accéder à la prévision.

M. VERRY souligne l'intérêt d'avoir mis en évidence, expérimentalement, que la transition pouvait faire reculer l'apparition de la cavité mais ajoute qu'il semble difficile d'exploiter le fait qu'on trouve par le calcul un décollement laminaire en amont d'une cavité dont le point de détachement est imposé.
En réponse à la question de $M$. LIESS concernant l'influence de la cavitation sur la fréquence des tourbillons dans le sillage d'un cylindre, M. MICHEL présente des résultats obtenus dans le cas d'un coin pour lequel l'effet de la cavitation sur les tourbillons alternés a été étudié.

Il trace la courbe du nombre de STROUHAL en fonction du nombre $\sigma$ et constate que l'allure de la courbe varie peu avec le REYNOLDS et qu'en dessous d'un certain $\sigma$ la cavitation est telle qu'il n'existe plus de sillage altemé.

En conclusion, il remarque que la cavitation a une dynamique propre réagissant considérablement sur le phénomène.

M. CASACCl clôt la discussion en insistant sur les progrès restant à faire dans la connaissance de la physique de ces phénomènes et sur l'importance de la liaison industrie-université.

\section{Abstract}

The cavitation developing on continuous-curve walls : the physics of detachment

During the 1970 s, a substantial research effort was carried out to explain the disparities traditionally described as "scale effects" found at the thresholds and on the figures of incipient cavitation; it revealed firstly the role played by the population of microbubbles of air in the water and secondly the relationship between the state of the boundary layer and the cavitation figures.

In the case of developed cavitation, the viscous effects are often overlooked. The present article describes recent experiments on continuous-curve bodies (circular and elliptical cylinders), which have however shown that an interaction exists between the boundary layer and the developed cavitation attached to a body. This interaction occurs in particular in the zone where the free boundary of the cavity becomes detached from the body: in this zone, the drop in ambient pressure leads to an extension of the cavitation and consequently to a change in the longitudinal pressure gradients that control the development of the boundary layer.

In the case of the circular cylinder, the position and direction of detachment was studied by statistical analysis of a large number of photographs. With regard to the position of the detachment, the results show strong influence of the Reynolds number alongside the cavitation parameter; they match to a large extent the more isolated results obtained by OBA, but show significant difference compared with ARAKERI's semi empirical modelization. The fast films show that the detachment zone is strongly affected by the nonpermanent nature of the wake close to the cylinder.

In the case of the two elliptical test bodies (with axis ratios $1 / 4$ and $1 / 8$ ), we examined in particular the following phenomena: the position of the points of detachment of the cavity, the forces (lift and drag) bearing on the body, and lastly the state of the boundary layer, visualized by injection of a coloured stream and calculated by the method developped at O.N.E.R.A. The pressure coefficients introduced into the calculation were themselves obtained from a purely hydrodynamic modelization using the method of discretized singularities. The parameters taken account of in this study were the cavitation and Reynolds numbers and the geometry of the profiles. The principal results wer as follows :

1) The infiuence of the Reynolds number on the detachment position was more significant in the case of the thickest profile. There is an angle of attack close to 3 degrees for which cavitation diseppears almost completely, when the cavitation number is moderate; at the same time the point of transition to turbulence moves further upstream. If the cavitation number is small, the cavity attaches itself to the profile in an almost unstable manner, depending on the three-dimensional configurations. At small angles of attack, detachment takes place behind the body and is preceded by laminary separation of the boundary layer. At larger angles, the cavity detaches close to the leading edge. It may be preceded either by transition or by laminary separation.

2) The lift coefficient is sensitive to the Reynolds number, especially when the angle of attack deviates from the ideal angle, for which the boundary layers develop symmetrically on the under and upper surfaces of the profiles.

3) Calculation of the boundary layer gives the point of laminary separation before detachment of the cavity in the cases of large or small angles of attack; it shows, in the cases of medium angles, the presence of transition to turbulence.

To sum up, this general study reveals that cavity detachment and the characteristic points of the boundary layer (laminary separation, the beginning of transition) develop analogously when the angle of attack of the body varies. 